\title{
EFECTO DEL EXTRACTO ETANÓLICO DE Calceolaria myriophylla “ZAPATILLA” SOBRE EL MODELO DEL SÍNDROME METABÓLICO INDUCIDO POR FRUCTOSA EN RATAS
}

\author{
Effect of ethanol extract of Calceolaria myriophylla "zapatilla" on the model of metabolic \\ syndrome fructosa induced in rats
}

\author{
Martín Condorhuamán ${ }^{1}$, Jorge L. Arroyo ${ }^{2}$, Oscar Herrera ${ }^{3}$, Luis A. Rojas ${ }^{1}$
}

${ }^{1}$ Instituto de Investigación en Ciencias Farmacéuticas y Recursos Naturales "Juan de Dios Guevara", Facultad de Farmacia y Bioquímica; ${ }^{2}$ Facultad de Medicina. Universidad Nacional Mayor de San Marcos. ${ }^{3}$ Facultad de Farmacia y Bioquímica, Universidad Nacional San Luis Gonzaga de Ica

\section{RESUMEN}

El presente estudio tuvo como objetivo determinar el efecto del extracto etanólico de Calceolaria myriophylla "zapatilla" en el síndrome metabólico inducido por fructosa en ratas. Se realizó el análisis fitoquímico cualitativo y se utilizó 48 ratas albinas machos de raza Holtzmann de 2 meses de edad con un peso que fluctuó entre 180 a 200 g. Se realizó la inducción del síndrome metabólico en ratas mediante la administración, vía oral, de fructosa al 6o\% durante 90 días y se trabajo según el siguiente diseño: seis grupos de ocho animales cada uno, divididos en grupo control, grupo fructosa, grupo fructosa más extracto etanólico (03 grupos a dosis de 200,400 y $600 \mathrm{mg} / \mathrm{kg}$, respectivamente y fructosa más medicamentos (atorvastatina $20 \mathrm{mg} / \mathrm{kg}$ y captopril $20 \mathrm{mg} / \mathrm{kg}$ en combinación); los tratamientos se administraron vía oral por 90 días. Se observo una disminución de los niveles de glucosa, perfil lipídico (colesterol total, HDL-colesterol y triglicéridos), presión arterial sistólica y diastólica y del peso corporal de las ratas en los grupos tratados. Se concluye que el extracto etanólico presentó efecto sobre los criterios clínicos y bioquímicos del síndrome metabólico inducido por fructosa en ratas que son estadísticamente significativos $(\mathrm{p}<0,05)$ y de la presencia en abundante cantidad de flavonoides y compuestos fenólicos.

Palabras clave: Calceolaria myriophylla, síndrome metabólico, fructosa.

\section{SUMMARY}

This study aimed to determine the effect of ethanol extract of Calceolaria myriophylla "shoe" in the metabolic syndrome induced by fructose in rats. Was made qualitative phytochemical analysis and used 48 male albino rats of race Holtzmann 2 months old with a weight fluctuated between 180 and $200 \mathrm{~g}$, was performed induction of metabolic syndrome in rats by oral administration of fructose to $60 \%$ for 90 days and work according to the following design: six groups of eight animals each group divided into control group, fructose group, fructose group more ethanolic extract (03 groups at doses of 200,400 and $600 \mathrm{mg} / \mathrm{kg}$, respectively and fructose more drugs (atorvastatin $20 \mathrm{mg} / \mathrm{kg}$ group and $20 \mathrm{mg}$ captopril $\mathrm{mg} / \mathrm{kg}$ in combination), treatments were administered orally for 90 days. Were observed decreased levels glucose, lipid profile (total cholesterol, HDL-cholesterol and triglycerides), systolic and diastolic blood pressure and body weight of rats in the treated groups. Was concluded that the ethanol extract showed effect on clinical and biochemical criteria on metabolic syndrome fructose induced in rats are statistically significant $(p<0,05)$ and the presence of abundant amounts of flavonoids and phenolic compounds.

Keywords: Calceolaria myriophylla, metabolic syndrome, fructose.

\section{INTRODUCCIÓN}

ए 1 síndrome metabólico (SM) se caracteriza por la presencia de alteraciones, como resistencia a la insulina, que se manifiestan por hiperinsulinismo y por su asociación con obesidad, diabetes mellitus tipo 2, hipertensión arterial y dislipidemia. La presencia de este síndrome se relaciona con incremento en el riesgo de aparición de enfermedades cardiocerebrovasculares y consecuentemente aumento de la mortalidad ${ }^{(1,2)}$.
El sobrepeso y la enfermedad cardiovascular continúa siendo la causa más común de muerte en los países industrializados, y la hipertensión arterial es el factor de riesgo tratable más frecuente. La prevalencia de hipertensión arterial en Europa es en promedio de $42,2 \%$, mientras que en Norteamérica es de $27,6 \%$. En la región de las Américas se considera que oscila entre el 10 y $25 \%$ de los adultos. En el Perú se ha reportado en el año 2004, en un estudio realizado en 26 ciudades, una prevalencia de hipertensión de $23,7 \%^{(3,4)}$. 
Tabla 1. Análisis fitoquímico cualitativo del extracto etanólico de Calceolaria myriophyll.

\begin{tabular}{lcc}
\multicolumn{1}{c}{ Reactivo } & Metabolito secundario & Resultado \\
\hline Magnesio + HCl (Shinoda) & Flavonoides & +++ \\
Cloruro férrico & Compuestos fenólicos & +++ \\
Hidróxido de sodio 5\% & Quinonas & + \\
Gelatina - sal & Taninos & + \\
Lieberman-Burchard & Triterpenoides & + \\
Prueba de la espuma & Saponinas & ++ \\
Naftol + ácido sulfúrico & Heterósidos & - \\
\hline
\end{tabular}

Leyenda ${ }^{11}$ : Abundante cantidad: +++ ; Regular cantidad: ++ ; Poca cantidad: + ; Ausencia: -

De acuerdo a un estudio realizado en el Perú, que usó los parámetros clínicos de la National Cholesterol Education Program ATP III (Adult Treatment Panel), en el departamento de Lambayeque, se encontró $28,3 \%$ de síndrome metabólico en mayores de 30 años de edad, $29,9 \%$ en el género femenino y en el masculino $23,1 \%$, siendo esta diferencia estadísticamente significativa. En Lima Metropolitana, en una población urbana de 30 a 92 años, se encontró 14,4\%; el 16,3\% en mujeres y $10 \%$ varones. Asimismo, en un estudio realizado en mujeres adultas con sobrepeso y obesidad, se comunicó una prevalencia de síndrome metabólico de 28 y $30 \%$, respectivamente ${ }^{(5)}$.

El tratamiento con drogas sintéticas previene más no cura la enfermedad, y conlleva a la presencia de reacciones adversas marcadas para cada grupo farmacológico como: estatinas que inducen a la rabdomiolisis, colestiramina que causa el aumento de la fosfatasa alcalina, exantema, urticaria y caída de pelo con los fibratos ${ }^{(6)}$. Por lo que se hace necesario realizar investigaciones con productos alternativos que sean eficaces y más seguros.

La Calceolaria myriophylla "zapatilla", es una hierba anual de 20 a $30 \mathrm{~cm}$. de altura, cuyas flores amarillas son parecidas a los zapatos, de ahí su nombre. Se encuentra en las laderas y cercos de piedra hasta los 4000 metros de altitud ${ }^{(7,8)}$.

Teniendo en cuenta las consecuencias y los riesgos que presentan las personas que presentan síndrome metabólico y los fármacos empleados, es necesario contar con productos naturales que ayuden en la profilaxis o el tratamiento de esta patología. Por lo tanto, la búsqueda de nuevos fármacos utilizando como fuente biológica los extractos de plantas medicinales, es un campo de plena vigencia sobre todo teniendo en cuenta las riquezas naturales de nuestro país, por lo cual se planteo el objetivo de determinar el efecto del extracto etanólico de Calceolaria myriophylla "zapatilla" en el síndrome metabólico inducido por fructosa en ratas.

\section{MATERIALES Y MÉTODOS}

Se colectó planta entera sin raíz de Calceolaria myriophylla en la comunidad de Q'orao, provincia del Cusco, región Cusco, a 3500 metros de altitud, en el mes de mayo. La muestra se sometió a secado en un horno a temperatura constante de $30^{\circ} \mathrm{C}$, realizando la

Tabla 2. Análisis de perfil lipídico, glucosa y pesos de los animales de experimentación.

\begin{tabular}{|c|c|c|c|c|c|c|c|}
\hline Tratamiento & Glucosa & $\begin{array}{l}\text { Colesterol } \\
\text { total }\end{array}$ & $\begin{array}{c}\text { HDL } \\
\text { - Colesterol }\end{array}$ & Triglicéridos & Peso inicial & Peso al mes & Peso final \\
\hline Control (agua destilada) & $87,8 \pm 2,31$ & $135 \pm 3,11$ & $43,8 \pm 1,63$ & $110,8 \pm 2,78$ & $213,4 \pm 9,99$ & $246,6 \pm 17,77$ & $267,2 \pm 8,82$ \\
\hline Control fructosa (F) & $113,2 \pm 1,82^{*}$ & $220 \pm 5,54^{*}$ & $29,8 \pm 2,08^{*}$ & $209,2 \pm 1,01^{*}$ & $264 \pm 5,86$ & $330 \pm 11,29$ & $345,6 \pm 9,53^{*}$ \\
\hline $\mathrm{F}$ - Extracto $200 \mathrm{mg} / \mathrm{kg}$ & $85,8 \pm 0,58$ & $122 \pm 5,38$ & $53,4 \pm 0,92$ & $103,2 \pm 0,66$ & $180,6 \pm 10,64$ & $215 \pm 16,70$ & $223,4 \pm 13,40$ \\
\hline $\mathrm{F}$ - Extracto $400 \mathrm{mg} / \mathrm{kg}$ & $82,2 \pm 3,08$ & $119,4 \pm 1,63$ & $54,2 \pm 0,37$ & $104 \pm 1,41$ & $159,8 \pm 5,77$ & $177,4 \pm 5,56$ & $203,6 \pm 4,16$ \\
\hline $\mathrm{F}$ - Extracto $600 \mathrm{mg} / \mathrm{kg}$ & $83,8 \pm 1,77$ & $139,2 \pm 1,77$ & $55 \pm 0,44$ & $96,8 \pm 1,24$ & $206 \pm 7,31$ & $241,2 \pm 4,95$ & $251,8 \pm 2,10$ \\
\hline F- Captopril + Atorvastatina $20 \mathrm{mg} / \mathrm{kg}$ & $87,8 \pm 4,06$ & $113,2 \pm 2,70$ & $52,8 \pm 0,86$ & $98,2 \pm 1,39$ & $294,8 \pm 19,04$ & $336,4 \pm 14,65$ & $328,2 \pm 15,97$ \\
\hline
\end{tabular}

F: fructosa. Los valores son expresados como media \pm error estándar. $\left({ }^{*}\right)$ Existe diferencia significativa entre las medias con respecto al grupo control fructosa $(p<0,05)$.

Tabla 3. Efecto hipotensor sobre la presión arterial sistólica y diastólica.

\begin{tabular}{|c|c|c|c|c|c|c|}
\hline Tratamiento & PBS & PBD & PS1 & PD1 & PS2 & PD2 \\
\hline Control (agua destilada) & $107,6 \pm 2,24$ & $88,6 \pm 2,33$ & $104,8 \pm 1,15$ & $82,8 \pm 2,74$ & $109 \pm 2,07$ & $80 \pm 3,20$ \\
\hline Control fructosa $(\mathrm{F})$ & $108,8 \pm 1,52$ & $86,6 \pm 3,77$ & $126,6 \pm 1,46^{*}$ & $103,6 \pm 0,67^{*}$ & $141 \pm 2,82^{*}$ & $119 \pm 2,88^{*}$ \\
\hline F - Extracto $200 \mathrm{mg} / \mathrm{kg}$ & $107,6 \pm 1,6$ & $83,8 \pm 2,22$ & $113,4 \pm 0,67$ & $90,2 \pm 6,28$ & $124,6 \pm 0,87$ & $103,6 \pm 0,67$ \\
\hline $\mathrm{F}$ - Extracto $400 \mathrm{mg} / \mathrm{kg}$ & $100,2 \pm 4,16$ & $78 \pm 2,70$ & $108 \pm 2,44$ & $82 \pm 2,70$ & $126,6 \pm 1,99$ & $102,4 \pm 2,13$ \\
\hline F - Extracto $600 \mathrm{mg} / \mathrm{kg}$ & $107,2 \pm 2,26$ & $79,8 \pm 3,51$ & $110,8 \pm 1,98$ & $83,2 \pm 3,85$ & $106,6 \pm 3,26^{* *}$ & $81,2 \pm 4,83^{* *}$ \\
\hline F - Captopril + Atorvastatina $20 \mathrm{mg} / \mathrm{kg}$ & $107,6 \pm 2,31$ & $79,2 \pm 2,41$ & $106,6 \pm 3,26$ & $81,2 \pm 4,83$ & $113,4 \pm 0,67(\mathrm{NS})$ & $90,2 \pm 6,28(\mathrm{NS})$ \\
\hline
\end{tabular}

Los valores son expresados como media \pm error estándar. PBS=Presión basal sistólica, PBD=Presión basal diastólica, PS1=Presión sistólica al mes, PD1=Presión diastólica al mes, PS2=Presión sistólica al final del estudio, PD2=Presión diastólica al final del estudio. $\left({ }^{\star}\right)$ Existe diferencia significativa entre las medias con respecto al grupo control fructosa $(p<0,05)$. $\left.{ }^{* *}\right)$ Existe diferencia significativa entre las medias con respecto al grupo de $200 \mathrm{y} 400 \mathrm{mg} / \mathrm{kg}(\mathrm{p}<0,05)$. (NS) No existe diferencia significativa entre las medias con respecto al grupo de $600 \mathrm{mg} / \mathrm{kg}$ y Captopril más Atorvastatina ( $p>0,05$ ). 
molienda en un molino eléctrico y luego tamizando con una malla para la uniformización del pulverizado. El extracto etanólico se obtuvo en una proporción del $20 \%$ $(\mathrm{P} / \mathrm{V})$, para lo cual se procedió a la maceración por un periodo de 15 días, y luego de filtrar se realizó el proceso de secado a una temperatura menor de $40^{\circ} \mathrm{C}^{(9,10)}$.

Se utilizaron 48 ratas albinas machos de raza Holtzmann, de 2 meses de edad, con un peso que fluctuó entre 180 a 200 gramos, obtenidos del bioterio del Instituto Nacional de Salud.

\section{Análisis fitoquímico}

Se realizó mediante pruebas fisicoquímicas de caracterización, mediante cambios de coloración o formación de precipitados ${ }^{(11)}$.

\section{Determinación del efecto sobre el síndrome metabólico inducido}

Se indujo el síndrome metabólico en ratas mediante la administración, vía oral, de fructosa al $60 \%$ durante 90 días ${ }^{(12)}$ y se trabajo según el siguiente diseño: seis grupos de ocho animales cada uno, divididos en grupo control, grupo fructosa, grupo fructosa más extracto etanólico (03 grupos a dosis de 200,400 y $600 \mathrm{mg} / \mathrm{kg}$ ) y grupo fructosa más medicamentos (atorvastatina $20 \mathrm{mg} / \mathrm{kg}$ y captopril $20 \mathrm{mg} / \mathrm{kg}$ en combinación) ${ }^{(13)}$, como ya se dijo, los tratamientos se administraron vía oral por 90 días.

Se evaluó el peso corporal de las ratas en balanza analítica al inicio y al término del tratamiento; el perfil lipídicoy la glicemia por métodos enzimáticosy los niveles de presión arterial sistólica y diastólica se midieron en forma incruenta en un equipo Letica $5002{ }^{(13)}$.

\section{Análisis estadístico}

Todos los datos fueron procesados en una base del paquete estadístico SPSS (Stadistical Package for Socials Sciences), versión 15.o en español. Se determinó la media y el error estándar del peso corporal, perfil lipídico, glicemia y la presión arterial comparando con los valores del grupo control, considerándose estadísticamente significativo con un $p<0,05$.

\section{RESULTADOS}

Los resultados se muestran en las tablas 1, 2 y 3.

\section{DISCUSIÓN}

En el análisis fitoquímico cualitativo (tabla 1) el extracto etanólico mostró la presencia de abundante cantidad de flavonoides y compuestos fenólicos y regular cantidad de saponinas, seguido de poca cantidad quinonas, taninos y triterpenoides. Estos resultados concuerdan con lo obtenido por Lastra y Yañez ${ }^{(14)}$, quienes determinaron la presencia de flavonoides, compuestos fenólicos, saponinas y taninos en el extracto seco hidroalcohólico de Calceolaria myriophylla.

El extracto etanólico presentó efecto sobre el peso corporal de las ratas, perfil lipídico y en la presión arterial sistólica y diastólica, siendo los datos estadísticamente significativos $(p<0,05)$, en relación al grupo control (tabla 2 y 3 ).

A la dosis de $600 \mathrm{mg} / \mathrm{kg}$ se evidenció mejor efecto hipotensor en relación a las dosis de 200 y $400 \mathrm{mg} / \mathrm{kg}$.

Existen múltiples reportes sobre el estudio de plantas medicinales para el tratamiento de la dislipidemia. Se debe señalar que el Plantago ovata (ispágula), Allium sativum (ajo) y Cynara scolymus (alcachofa), han sido reportados por presentar efecto hipocolesterolemiante ${ }^{(15)}$; la actividad hipocolesterolemiante de la hoja de alcachofa se atribuye a diferentes compuestos, como las lactonas sesquiterpénicas (cinaropicrina, grosheimina), y los flavonoides (luteolina, glucósidos de luteolina) (16). La administración del extracto acuoso de Aloe vera en conejos, durante 6 semanas, produjo una disminución de los niveles de lípidos en suero y un aumento del HDL-colesterol, lo que es considerado como un efecto beneficioso asociado a la disminución de infarto ${ }^{(17)}$.

El estudio de extractos de plantas medicinales para el tratamiento de la hipertensión arterial, desarrollado en modelos preclínicos y clínicos, muestran metabolitos fitoquímicos activos, como son los flavonoides, compuestos fenólicos y saponinas esteroidales. El mecanismo de acción antihipertensivo de los dos compuestos señalados serían por un efecto vasodilatador e inhibición del sistema renina angiotensina, asimismo, el efecto diurético puede tener como responsable a la presencia de flavonoides y compuestos fenólicos ${ }^{(18)}$, los que explican que dicho efecto se debería a la acción vasodilatadora, osmótica, inhibición del sistema renina angiotensina aldosterona y de la reabsorción de sodio ${ }^{(19)}$, contribuyendo de esta manera a la disminución de la presión arterial.

\section{CONCLUSIONES}

El extracto etanólico de Calceolaria myriophylla presentó efecto sobrelos criterios clínicosy bioquímicos del síndrome metabólico inducido por fructosa en ratas, siendo) estadísticamente significativos $(p<0,05)$; 
y se identifico la presencia en abundante cantidad de flavonoides y compuestos fenólicos.

\section{REFERENCIAS BIBLIOGRÁFICAS}

1. Barrera M, Pinilla A, Cortés E, Mora G, Rodríguez M. Síndrome metabólico: una mirada interdisciplinaria. Rev Col Cardiol 2008; 15(3): 111-26.

2. Comós JB, Murillo M. Obesidad y síndrome metabólico. Protoc Diagn Ter Pediatr 2011; 1: 228-35.

3. McGill HC, McMahan CA, Zieske AW, Tracy RE, Malcom GT, Herderick EE, et al. Association of coronary heart disease risk factors with microscopic qualities of coronary atherosclerosis in youth. Circulation 2000; $102(4)$ : 374-9.

4. Wolf-Maier K, Cooper R, Banegas J, Giampaoli S, Hense $\mathrm{H}$, Joffres $\mathrm{M}$, et al. Hypertension prevalence and blood pressure levels in 6 European countries, Canada and United States. JAMA 2003; 289(18): 2363-9.

5. Pajuelo J, Sánchez J. El Síndrome metabólico en adultos, en el Perú. An Fac Med 2007; 68(1):38-46.

6. Agusti R. Epidemiología de la hipertensión arterial en el Perú. Acta Med Per. 2006; 23(2): 69-75.

7. Olazábal C, Mantillas J. Las Plantas Medicinales de Nuestra Tierra. Instituto de Ecología y Plantas Medicinales - IEPLAM. Cusco, 2001.

8. Roesrch C. Plantas Medicinales en el Sur Andino del Perú. Tomo 2. Koeltz Scientific Books. Centro de Medicina Andina (CEMA). Cusco, 1994.

9. CYTED. Búsqueda de principios activos de planas de la región. Manual de técnicas de investigación. Programa Iberoamericano de Ciencia y Tecnología para el Desarrollo. 1995.

10. Sharapin N. Fundamentos de tecnología de productos fitoterapéuticos. Publicación del programa de Andrés Bello - Programa Iberoamericana de Ciencia y Tecnología para el Desarrollo. Santa Fe de Bogotá, 2000.

11. Lock De Ugaz O. Investigación Fitoquímica - Métodos en el estudio de Productos Naturales. Fondo Editorial de la Pontificia Universidad Católica del Perú. Lima, 1988.
12. De Moura RF, Ribeiro C, De Oliveira JA, Stevanato E, De Mello MA. Metabolic syndrome signs in Wistar rats submitted to different high-fructose ingestion protocols. Br J Nutr 2009; 101(8): 1178-84.

13. Arroyo J, Rojas J, Chenguayen J. Manual de Modelos Experimentales de Farmacología. ASDIMOR. Lima, 2004 .

14. Lastra Y, Yáñez B. Efecto gastroprotector de Gamochaeta americana (Mill) Wedell (K'eto K'eto) y Calceolaria myriophylla Kraenz (Ayaq Zapatilla) sobre lesiones gástricas inducidas por indometacina en ratas. [Tesis para Optar al Título Profesional de Químico Farmacéutico]. Facultad de Ciencias Químicas, Físicas, Matemáticas, Farmacia e Informática. Universidad Nacional San Antonio Abad del Cusco. Cusco, 2004.

15. López T. Plantas medicinales para el tratamiento de la hipercolesterolemia. Fitoterapia 2002; 21(6): 138-44.

16. Villar A, Abad M. Hojas de alcachofa: indicaciones terapéuticas. Farmacia Profesional 2004; 18(11): 58-61.

17. Tedesco D, Tava A, Galletti S, Tameni M, Varisco G, Costa A, et al. Effects of silymarin, a natural hepatoprotector, in periparturient dairy cows. J Dairy Sci 2004; 87(7): 223947 .

18. Kuklinski C. Farmacognosia. Estudio de las drogas y sustancias medicamentosas de origen natural. Omega SA. Barcelona, 2003.

19. Maghrani M, Zeggwagh A, Haloui M, Eddouks M. Acute diuretic effect of aqueous extract of Retama raetam in normal rats. J Ethnopharmacol 2005; 99(1): 31-5.

Manuscrito recibido el: 07/10/14

Aceptado para su publicación el: 20/10/14

\section{Correspondencia:}

Nombre: $\quad$ Martín Condorhuaman Figueroa

Dirección: Jr Puno 1002 Lima

e-mail: marcofin1@yahoo.es 